\title{
Incorporación de la mujer al mercado de trabajo y desarrollo regional en Chihuahua
}

\author{
Luis Enrique Gutiérrez Casas* \\ y Myrna Limas Hernández**
}

\begin{abstract}
Resumen. Este ensayo aborda la relación entre el desarrollo y la incorporación de la mujer al mercado de trabajo. De manera específica se revisan, bajo métodos estadísticos, los vínculos entre las tasas de participación económica femenina [TPF] y variables asociadas al desarrollo, como la estructura económica, el nivel de ingreso, la pobreza, la desigualdad, la marginación, el desarrollo humano, así como algunos indicadores de género. El caso que se examina en este trabajo es el de los municipios del estado de Chihuahua (México) y con base en el análisis empírico se plantea un marco de relaciones de causalidad entre las TPF y el desarrollo de las regiones. Este estudio concluye que la participación femenina en la economía tiene un impacto relevante en el desarrollo, por lo que es preciso la formulación y aplicación de políticas que otorguen mayor capacidad y amplíen las oportunidades para la incorporación de la mujer al mercado laboral.

Palabras clave: tasas de participación femenina, género, desarrollo, desarrollo regional, Chihuahua.
\end{abstract}

\begin{abstract}
This paper explores the relationship between economic development and the entrance of women into the labor market. In particular are reviewed the links between female labor force participation [FLFP] and several variables associated to development -economic structure, income level, poverty, inequality, marginalization, human development and other specific gender indicators. This essay analyses the case of the municipalities of the mexican northern state of Chihuahua. Based on the empirical study it is proposed a causal relationship framework between the FLFP and the development of regions. This paper concludes that women participation in the economy has a significant impact on development; therefore, regions must formulate public policies in order to achieve more opportunities for the incorporation of women into the labor market.

Keywords: female labor force participation, gender, development, regional development, Chihuahua.
\end{abstract}

* Profesor del área de economía del Departamento de Ciencias Sociales de la Universidad Autónoma de Ciudad Juárez. Correo electrónico: lgtzc@yahoo.com; lgtz@uacj.mx

** Profesora del área de economía del Departamento de Ciencias Sociales de la Universidad Autónoma de Ciudad Juárez. Correo electrónico: mlimas@uacj.mx 


\section{Presentación}

El desarrollo de las regiones y la incorporación de la mujer al mercado de trabajo se encuentran estrechamente asociados. En lo general se puede afirmar que conforme una mayor proporción de mujeres se integra a los sectores productivos de la economía, un conjunto de distintas variables vinculadas al desarrollo regional -desde algunas estrictamente económicas hasta otras de carácter socioeconómicomuestran un comportamiento positivo, signo de una mejor calidad de vida de la población. No obstante, la relación de causalidad entre la participación de la mujer en la actividad productiva ${ }^{1}$ y el nivel de desarrollo es compleja; no es unidireccional o lineal y, en todo caso, podría entenderse como circular o, como lo expresan Pampel y Tanaka (1986), "interactiva"; esto es, en tanto el mismo proceso de desarrollo estimula una participación femenina creciente en el mercado laboral, ésta retroalimenta y fomenta el desarrollo.

El presente ensayo es básicamente un ejercicio empírico que, mediante métodos estadísticos, intenta confirmar, para el caso particular del estado fronterizo de Chihuahua (México), la asociación entre la participación de la mujer en las economías locales con el comportamiento de otras variables ligadas al desarrollo de las regiones. Cabe decir que aquí no se hace referencia a especificidades de la incorporación de la mujer en el mercado laboral conforme a las distintas líneas temáticas y disciplinarias de los estudios de género y los mercados laborales, tal como se clasifican en otros estudios (Martínez, 2001). ${ }^{2}$ Este trabajo, en todo caso, pretende constatar, en el contexto regional

\footnotetext{
${ }^{1}$ En la economía de género se reconocen dos tipos de trabajos esenciales para el funcionamiento de una economía: el productivo y el reproductivo. El trabajo productivo, al que hacemos referencia en este ensayo, se define como el trabajo remunerado dirigido a producir bienes y servicios para el mercado; el trabajo reproductivo se entiende como el conjunto de actividades domésticas y de cuidado familiar no remuneradas.

${ }^{2}$ Martínez hace referencia a una serie de temas específicos que pueden abordarse en los estudios sobre la participación femenina en los mercados de trabajo. Estos temas pueden ir desde el acoso sexual o las formas de control de hombres sobre las mujeres en los centros de trabajo, hasta los riesgos laborales, la salud y la reproducción, o la discriminación y la seguridad laboral, entre otros.
} 
del estado de Chihuahua, una serie de relaciones básicas entre el nivel de desarrollo y lo que se ha denominado la feminización de los mercados de trabajo.

Como parte de este ejercicio se analizan y relacionan la tasa de participación femenina y el índice de feminización de la población económicamente activa en el estado de Chihuahua con el crecimiento económico de la misma entidad, los cambios en su estructura sectorial y un conjunto de indicadores socioeconómicos del desarrollo, entre los que destacan los índices de pobreza, desigualdad, marginación y de desarrollo humano. El diagrama 1 esquematiza los vínculos entre la feminización de los mercados laborales y el proceso de desarrollo bajo las premisas asumidas en este ensayo. ${ }^{3}$

El texto está organizado en cinco apartados. El primero explica una serie de posiciones teóricas entre desarrollo y género; el segundo describe el proceso de feminización de la fuerza de trabajo en el estado de Chihuahua y examina la asociación entre el nivel de ingreso y la estructura sectorial con las tasas de participación femenina; el tercero describe el vínculo entre las TPF y los niveles de pobreza, desigualdad, marginación, desarrollo humano y algunos indicadores de género; el cuarto propone una serie de relaciones causales entre el desarrollo y la incorporación de la mujer al mercado de trabajo; $y$, finalmente, el quinto apartado presenta las conclusiones del ensayo.

Cabe decir que la información se revisó a escala municipal para los años 1990 y 2000. Con base en este último año se hacen las principales inferencias debido a que i) la información es más completa respecto a las variables utilizadas y ii) los datos nos proporcionan un marco de referencia más amplio respecto al nivel de desarrollo del estado y la participación femenina en la economía. Es preciso añadir que en el apartado cuatro se presenta una síntesis de los resultados de las me-

${ }^{3}$ Para efectos de este trabajo se interpreta que el crecimiento es una condición necesaria pero no suficiente para el desarrollo, mientras el desarrollo es indispensable para el crecimiento sostenido de una economía, en este caso regional. Ambas nociones, por tanto, son parte de un mismo proceso circular que tiende a propiciar un más elevado nivel de vida a la población. En este sentido, en ocasiones parecerá que los conceptos crecimiento y desarrollo se utilizan como sinónimos; no obstante, debe aclararse que en todo momento se tiene presente la diferencia de estos dos términos desde la perspectiva cuantitativa y cualitativa. 
Diagrama 1.

Vínculo entre participación de la mujer en el mercado de trabajo y el desarrollo

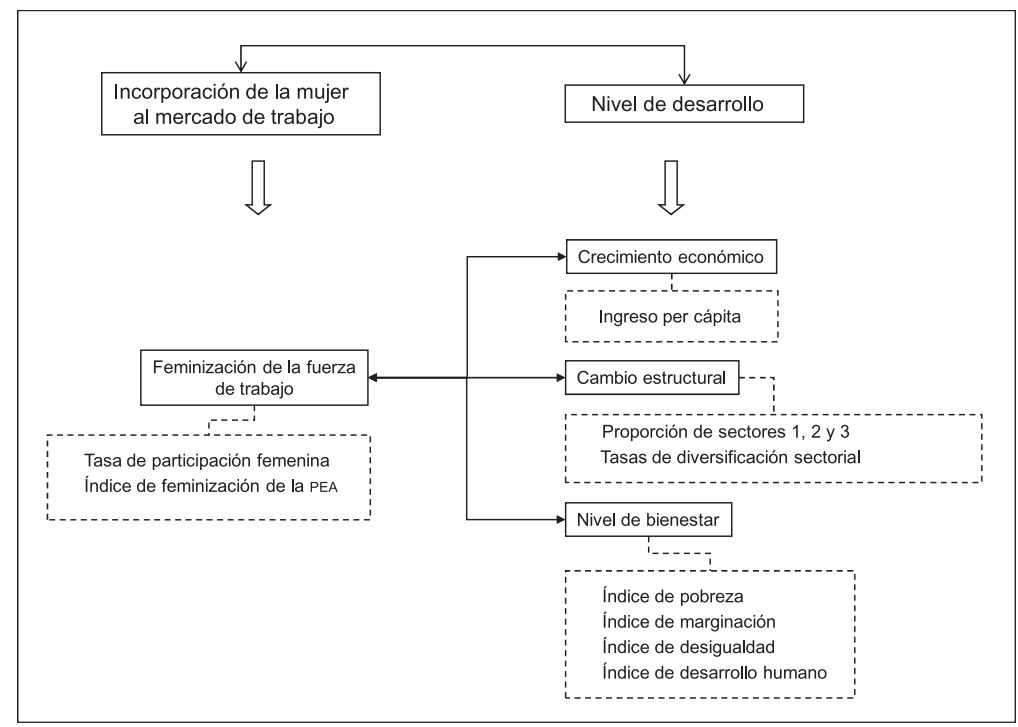

Fuente: Elaboración propia.

diciones estadísticas que formaron parte de este trabajo, los que a su vez nos permiten confirmar el vínculo entre la incorporación de la mujer al mercado laboral y el desarrollo regional.

\section{Consideraciones teóricas}

La modernización económica y el cambio estructural son manifestaciones del desarrollo que no se expresan en forma neutral en términos de género. Desde hace varias décadas, trabajos pioneros como el de Boserup (1970) abordaron de manera integral el impacto del desarrollo económico en la mujer, ${ }^{4}$ de modo que desde la misma disciplina económica

${ }^{4}$ Esther Boserup escribe que el desarrollo económico y social conlleva inevitablemente la desintegración de la división tradicional del trabajo entre hombre y mujer. 
han proliferado y evolucionado un conjunto de teorías y estudios empíricos sobre la participación femenina en la economía (Barker, 2005; Benería, 2003a), mientras que desde la perspectiva de las políticas públicas el papel de la mujer como agente del desarrollo se ha revisado a partir de distintas vertientes (Momsen, 2006). ${ }^{5}$

En particular la relación entre la incorporación de la mujer al mercado de trabajo y el desarrollo económico puede analizarse bajo perspectivas diversas. Sin embargo, dentro de la literatura económica sobresale un conjunto de estudios que explora el vínculo entre el desarrollo en general, el proceso de industrialización -o, en su caso, el grado de diversificación sectorial de países y regiones- y la tasa de participación femenina [TPF] en países y regiones. Para el caso que nos ocupa, la TPF se define, en términos generales, como el porcentaje de mujeres de 12 años y más que forman parte de la población económicamente activa femenina $\left[\mathrm{PEA}_{\mathrm{f}}\right]{ }^{6}$

A partir de una serie de trabajos que analizan las tasas de participación femenina y el cambio económico, se han formulado tres hipótesis o posiciones que compiten entre sí ante el intento de definir y pronosticar la pauta de comportamiento de las TPF conforme una economía transita diferentes niveles de desarrollo (Rau y Wazienski, 1999). La primera hipótesis, la más generalizada, plantea que las TPF tienen un comportamiento en forma de $U$ (curvilineal); la segunda, a la que se le denomina hipótesis de la emancipación, predice una relación directa (lineal); finalmente, la tercera hipótesis explica que las TPF son constantes, independientemente del grado de desarrollo de una economía (véase gráfica 1).

${ }^{5}$ Lourdes Benería expresa que la "economía feminista ha elaborado una agenda que va más allá del análisis convencional del capital humano y la fuerza laboral, con el fin de reclamar igualdad de oportunidades a la vez que enfatizar la importancia de los derechos productivos, políticas de familia, economía del cuidado y políticas contra la discriminación" (Benería, 2003b:42).

${ }^{6}$ Bajo los criterios de la ONU y la Organización Internacional del Trabajo (OIT), la población en edad de trabajar es de 15 años y más. No obstante, en el caso de México se toma en cuenta a la población femenina de 12 años y más (INEGI). La notación formal se expresa como:

$$
T P F=\frac{P E A_{f}}{P T_{f 12+}} \times 100
$$


Gráfica 1.

Nivel de desarrollo y tasas de participación femenina Hipótesis de comportamiento de la TPF

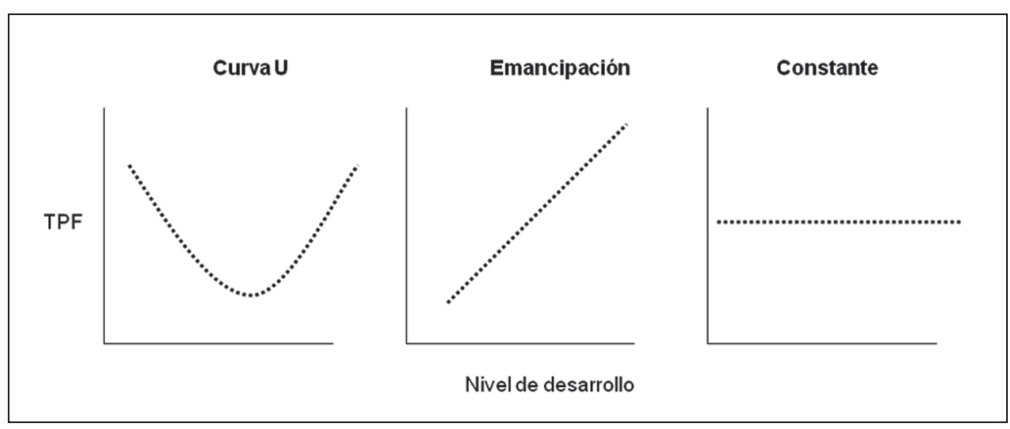

Fuente: Elaboración propia con base en Rau y Wazienski, 1999.

La hipótesis de la "curva U" o relación curvilineal, conocida como la hipótesis Singh-Goldin-Duran, predice que el crecimiento económico es inicialmente acompañado por una reducción en las TPF, las cuales después de llegar a un punto mínimo- se recuperan y alcanzan niveles más elevados en la medida en que el crecimiento se sostiene. La lógica de esta posición la podemos encontrar en un trabajo ya clásico de Mammen y Paxson, quienes sostienen que

[...] puede haber una relación en forma de $U$ entre la participación de la fuerza de trabajo femenina y el desarrollo económico. En naciones muy pobres, la participación de la fuerza de trabajo femenina es alta, y las mujeres trabajan en empresas familiares agropecuarias y no-agropecuarias. Inicialmente, el desarrollo desplaza a las mujeres de la fuerza de trabajo, en parte por el incremento de oportunidades para los hombres en el mercado de trabajo, y en parte por las barreras sociales que las mujeres enfrentan para incorporarse a la fuerza laboral asalariada. Sin embargo, a medida que las naciones continúan el proceso de desarrollo, los niveles de educación de la mujer aumentan y las mujeres se reincorporan a la fuerza de trabajo como empleadas asalariadas, manteniendo principalmente empleos de cuello blanco (Mammen y Paxson, 2000:144). 
Aun cuando el trabajo mencionado de Mammen y Paxson, así como los de Pampel y Tanaka (2001) o Rau y Wazienski (1999) han confirmado con distintos matices la hipótesis de la curva $U$ en el caso de varios países y regiones, cabe señalar que otros estudios la han descartado como único patrón de comportamiento entre las TPF y el desarrollo económico (Dureya, 2001; Abramo y Valenzuela, 2005).

En cuanto a la hipótesis de la emancipación, ésta sugiere una relación directa entre el proceso de industrialización, el crecimiento del empleo femenino y la libertad de la mujer (Shorter, 1973). La idea principal de este planteamiento es que históricamente las economías han pasado de una situación preindustrial y patriarcal a economías industriales y posindustriales con mercados impersonales que promueven una nueva mentalidad en la mujer joven, lo cual le permite trascender el ámbito del trabajo doméstico. De este modo, a medida que una economía escala niveles de desarrollo superiores, se espera que la incorporación de la mujer en los mercados laborales se eleve.

Finalmente, la hipótesis de la TPF constante sostiene que la mujer siempre ha trabajado, independientemente del nivel de desarrollo, pero la estadística oficial ha cometido errores de subconteo al no considerar una gran variedad de actividades remuneradas y no remuneradas de la mujer, o incluso las ha omitido. En otras palabras, esta hipótesis sugiere que el trabajo de la mujer se encuentra devaluado (Bose, 1987), lo que conlleva una subestimación del papel de la mujer en la economía. Estudios como el de Benería (1981) han dado cuenta detallada del sesgo ideológico y los problemas de contabilización que tienen como consecuencia el cálculo de TPF menores a las que efectivamente se presentan en una economía.

Cabe añadir que la literatura económica también cuenta con trabajos que plantean una serie de determinantes o factores que influyen en las TPF y, por lo tanto, en el grado de participación de la mujer en los mercados de trabajo. Entre estos determinantes sobresalen el desarrollo industrial, el nivel educativo y las oportunidades de formación de la mujer, la fertilidad, la desigualdad de ingreso, el papel del Estado, el nivel de inversión extranjera en una economía y el tipo de especialización, así como los ciclos económicos y la expansión de la fuerza laboral, la cultura y los contextos históricos. La peculiaridad 
de estos determinantes es que no muestran un comportamiento causal lineal, sino "interactivo" entre sí (Pampel y Tanaka, 1986).

Es preciso aclarar que aunque este estudio revisa estrictamente el vínculo de las TPF y una serie de variables de corte económico referidas al desarrollo, ello no implica aceptar que la incorporación de la mujer al mercado laboral no propicia cambios en otras esferas de la vida social o, incluso, el tema se limite a ser abordado desde la perspectiva del análisis económico. Trabajos como los de Smith y Ward (1989) o Pickford (1976) advirtieron desde hace varios años de los cambios que tendría la estructura familiar, por ejemplo, al incorporarse una proporción mayor de mujeres al mercado de trabajo.

Independientemente de las diferentes propuestas teóricas, varios autores coinciden en un comportamiento claro y sostenido de las tasas de participación femenina a escala mundial y la feminización de la fuerza de trabajo. Chihuahua no es la excepción y este proceso ha sido patente en las últimas décadas, particularmente en los años noventa. En los siguientes dos apartados se abordan algunos aspectos generales de este proceso en el estado. Cabe decir que, en lo general, el impacto que tiene el desarrollo económico en las TPF, así como la influencia positiva que el incremento de estas mismas tasas ejerce sobre el desarrollo ha sido explorado en trabajos como los de Shultz (1990), Maxwell (1990) y Michael (1985). ${ }^{7}$

\section{Nivel de ingreso, estructura económica y feminización de la fuerza de trabajo en Chihuahua}

\section{Feminización de la fuerza de trabajo}

La tendencia mundial en los mercados laborales se define, desde la perspectiva de género, por la feminización de la fuerza de trabajo. ${ }^{8}$

\footnotetext{
${ }^{7}$ En un reciente trabajo coordinado por Loutfi (2006) se puede encontrar una amplia variedad de temas que en la actualidad forman parte de la discusión sobre género y desarrollo.

${ }^{8}$ Dentro de la economía de género también se habla de procesos de "doble feminización", que por un lado refieren "el número creciente de mujeres vincula-
} 
Este fenómeno implica una importancia relativa creciente -y en ocasiones preponderante- de las mujeres en la estructura ocupacional de las economías regionales. La incursión de una proporción cada vez más significativa de mujeres en distintos sectores productivos se manifiesta como una pauta consistente desde hace dos décadas, y es una característica distintiva de diferentes experiencias de desarrollo que en la actualidad han resultado en un mayor equilibrio de género en la población económicamente activa, así como en tasas de participación femenina más elevadas (Abramo y Valenzuela, 2005). ${ }^{9}$

El estado de Chihuahua ha replicado este proceso de feminización de los mercados de trabajo. La TPF de la entidad pasó de 23 a 34\% en el periodo 1990-2000 (un incremento del 45\%), en tanto la tasa de participación masculina, que inició con $71 \%$, terminó en $72 \%$ (un incremento mínimo para el mismo periodo). Como puede apreciarse en la gráfica 2, las TPF por municipio también muestran variaciones positivas claramente mayores respecto a las tasas de participación masculina [TPM]. Estas últimas, cuando no arrojaron decrementos, simplemente mostraron un crecimiento no significativo. En el caso de la gráfica 2, los municipios se ordenaron de menor a mayor nivel de ingreso per cápita en 2000, a partir de lo que se puede observar, como pauta general, que la mayor variación en las TPF durante el periodo de referencia (1990-2000) ocurrió en los municipios con menor nivel de ingreso (gráfica 2a). ${ }^{10}$

Además, si utilizamos el índice de feminización de la población económicamente activa [IFP], ${ }^{11}$ también podremos constatar que el nú-

das a los sistemas de producción [...] y, por otra parte, alude a la configuración de condiciones de empleo más inestables, menos seguras, más temporales, peor pagadas, es decir, propias de los rasgos tradicionales asignados al empleo de las mujeres a lo largo de la historia en la mayor parte de las economías" (Vara, 2006).

${ }^{9}$ Un trabajo más completo para el caso de México se puede ver en Cardero (2003).

10 Trabajos como los de Jacobsen (1998) hacen un intento por analizar las causas que explican las tendencias crecientes y decrecientes de las tasas de participación económica femenina y masculina.

${ }^{11}$ El índice de feminización al que hacemos referencia expresa el número de mujeres que participan en la PEA en relación a cada hombre que también forma parte de la misma. La notación formal es:

$$
I F P=\frac{P E A_{f}}{P E A_{m}}
$$


Gráfica 2.

Variación de las tasas de participación económica femenina y masculina por municipio según nivel de ingreso.

Chihuahua, 1990-2000

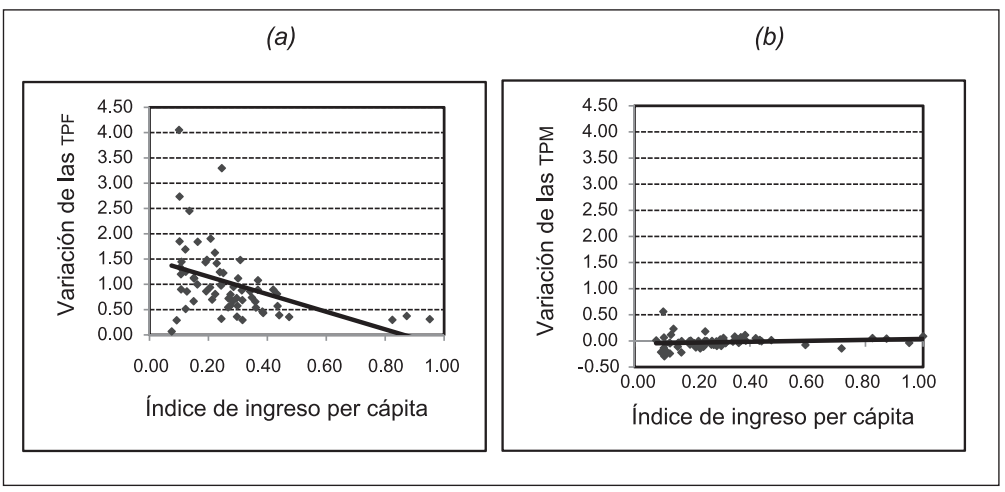

Fuente: Elaboración propia con base en Conapo (2001a) e INEGI (1990, 2000).

Nota: Debido a que muestran valores atípicos no congruentes con otra información disponible respecto al nivel de ingreso, no se consideran los municipios de Galeana, Gómez Farías y Matachí. Esta misma aclaración procede para las siguientes gráficas en las que se utiliza el índice de ingreso per cápita.

mero de mujeres dentro del mercado de trabajo, en relación con cada hombre, se incrementa de 1990 a 2000. En Chihuahua, el IFP pasó de 0.35 a 0.49 . En el caso de la gráfica 3 , en el eje de las abscisas los municipios se encuentran nuevamente ordenados según el nivel de ingreso per cápita. La revisión de las gráficas $3 a$ y $3 b$ nos permite apreciar un incremento general del IFP en los municipios de la entidad. Asimismo, la relación entre índice de ingreso per cápita [IPC] y el IFP nos indica que el índice de feminización se incrementa a medida que los municipios tienen un mejor nivel de ingreso y, por lo tanto, muestran un nivel mayor de desarrollo.

Con ambos indicadores, la TPF y el IFP, se constata que durante los años noventa se presentó un proceso de feminización de la fuerza de trabajo en todo el estado. Y más aún, se aprecia el estrecho vínculo que guardan los cambios positivos en las tasas de participación femenina y el índice de feminización de la PEA con variables asociadas al 
desarrollo, como lo son el nivel de ingreso y la estructura económica. A continuación revisamos con más detalle esta relación.

\section{El nivel de ingreso y la estructura económica}

La literatura económica indica que el nivel de desarrollo se vincula al nivel de ingreso per cápita, así como a los niveles de pobreza y marginación (Ray, 1998). En el caso del estado de Chihuahua esta relación ha quedado demostrada en trabajos como el de Gutiérrez (2007). En este sentido, podemos entender que diferentes niveles de ingreso per cápita son a su vez diferentes grados de desarrollo y, por lo tanto, de bienestar. Asimismo, las tasas de participación femenina se encuentran asociadas al nivel de desarrollo. La gráfica 4 muestra la relación directa entre TPF e ingreso per cápita. Esta asociación indica que para los municipios de Chihuahua la incorporación de la mujer al mercado de trabajo sí está asociada positivamente a un nivel de ingreso per cápita más elevado. Cabe observar que los dos municipios con mayor nivel de ingreso muestran un techo de participación femenina del $40 \%$.

\section{Gráfica 3.}

Índice de feminización de la PEA por municipio según nivel de ingreso per cápita.

Chihuahua, 1990-2000

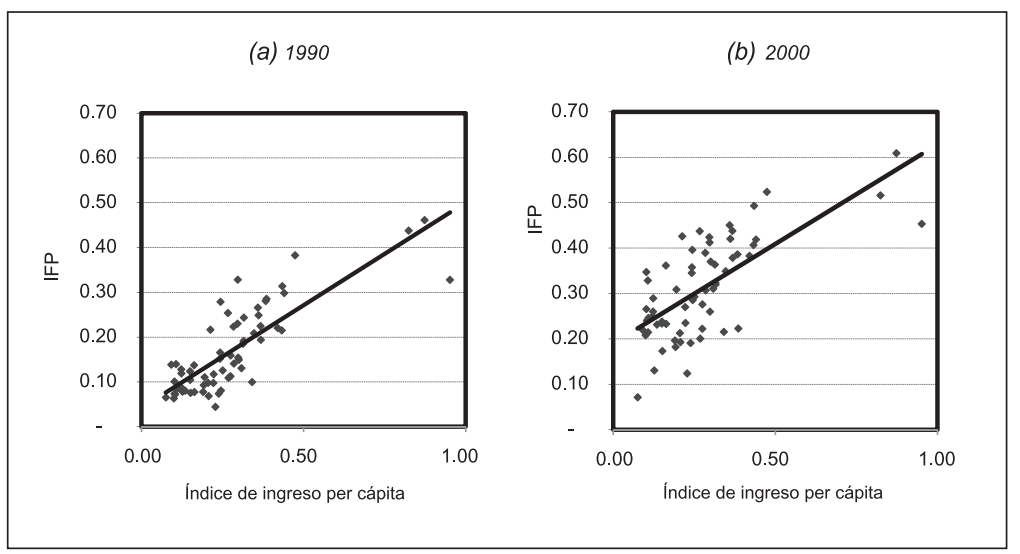

Fuente: Elaboración propia con base en Conapo (2001a) e INEGI $(1990,2000)$. 
Gráfica 4.

Tasas de participación femenina por municipio según nivel de ingreso per cápita.

Chihuahua, 2000

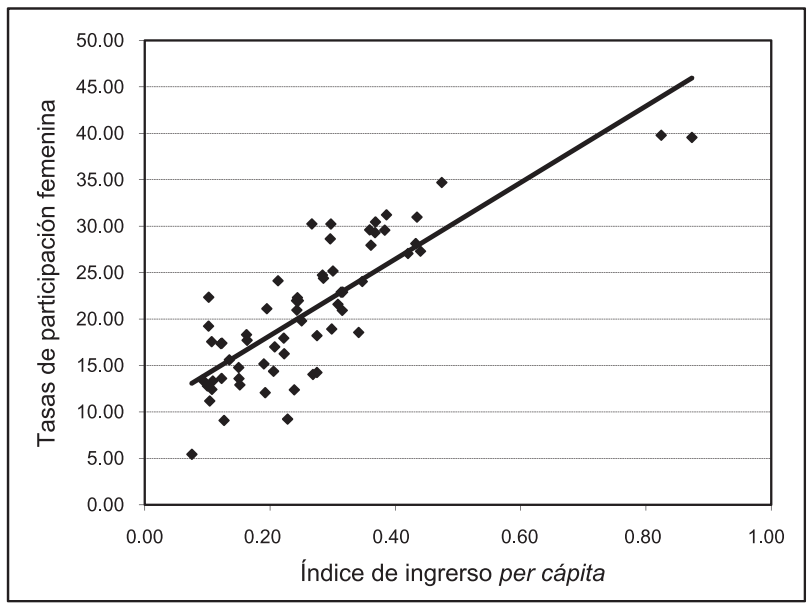

Fuente: Elaboración propia con base en Conapo (2001a) e INEGI (2000).

Por su parte, la relación entre estructura económica y crecimiento económico ha sido ampliamente fundamentada. Los trabajos de Kuznets, por ejemplo, dejan claro que las economías atrasadas están relacionadas con una alta dependencia hacia el sector primario; en tanto las economías con tasas de crecimiento mayores son aquellas que tienen una importante representación industrial o, más aún, manifiestan un dinámico y flexible sector terciario. En tal sentido, la diversificación de la estructura económica (cambio estructural) es un componente necesario para la expansión económica y, por ende, el desarrollo. Kuznets también sostiene que el crecimiento es tan sólo una manera "de transferir trabajo desde la 'atrasada' agricultura a la 'progresiva' industria" (Kuznets, 1970; 40). No obstante, conforme a este mismo autor, las transformaciones estructurales no son únicamente de carácter sectorial, expresadas en una creciente industrialización, sino también se resumen en procesos de urbanización y cambios en la dinámica demográfica, en la organización económica y hasta en 
los valores sociales. Un cambio estructural es, a fin de cuentas, una "adaptación interna" al potencial de crecimiento.

En el caso de Chihuahua, las transformaciones estructurales han ido más allá del cambio sectorial y han tenido incidencia tanto en el ingreso como en la dinámica laboral, desde la perspectiva de género. Los cambios en la composición por sectores productivos de las economías municipales muestran una estrecha relación con el grado de desarrollo de los municipios y, en este caso, con la incorporación de la mujer al mercado de trabajo no agropecuario. Esto es, hay una asociación directa entre la diversificación sectorial, el nivel de ingreso y las tasas de participación femenina. Entre mayor es la representación de los sectores secundario y terciario respecto a las actividades agropecuarias, mayor es la TPF y, por lo tanto, mayor es el ingreso per cápita. ${ }^{12}$

En la gráfica 5 se aprecia la relación entre ingreso per cápita y estructura sectorial en el estado. Como se puede observar, a mayor proporción de los sectores secundario y terciario en la economía -y por ello un mayor grado de diversificación-, el nivel de ingreso aumenta. En la gráfica 6 se revela asimismo la estrecha asociación que existe entre la estructura ocupacional por sector productivo y las tasas de participación femenina. Las gráficas $6 \mathrm{a}$ y $6 \mathrm{~b}$ muestran la asociación directa entre estructura económica y TPF, en tanto la gráfica $6 \mathrm{c}$-por lógica- muestra una relación inversa entre el tamaño del sector primario y las tasas de participación femenina. En todo caso, los datos que aquí se muestran señalan que una mayor participación de la mujer en los sectores secundario y terciario se encuentra asociada a un nivel de ingreso creciente.

Siguiendo con el análisis, también puede apreciarse que un mayor dinamismo en la estructura económica de las economías municipales se vincula con la incorporación más activa de las mujeres al mercado de trabajo en los sectores secundario y terciario. En la gráfica $6 \mathrm{~d}$ se relacionan la tasa de crecimiento sectorial conjunta de los sectores se-

12 Debe anotarse que, aun con estos cambios, el propio estado de Chihuahua experimenta serios desequilibrios territoriales y socioeconómicos, que se concretan en la existencia de un grupo mayoritario de municipios atrasados y menos desarrolados vinculados al sector agropecuario, y un conjunto de municipios más desarrollados y con una estructura económica más diversificada (Gutiérrez, 2007). 
Gráfica 5.

Estructura sectorial e ingreso per cápita por municipio Chihuahua,2000

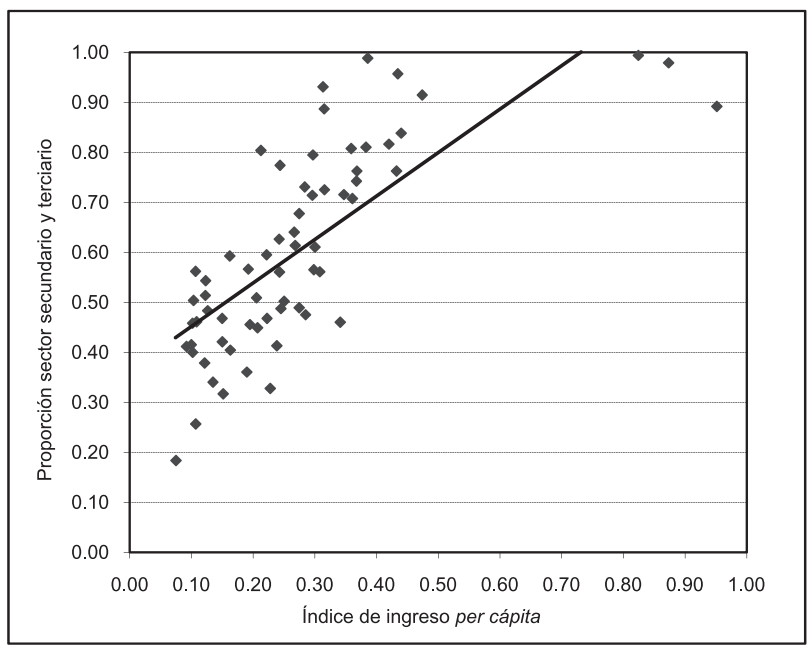

Fuente: Elaboración propia con base en CONAPO (2001a) e INEGI (2000).

cundario y terciario con la tasa de variación de la TPF a escala municipal. El resultado es claro: los municipios con mayor diversificación económica resultaron también con una incorporación femenina más dinámica.

\section{Las variables socioeconómicas y las tasas de participación femenina}

En este apartado intentaremos asociar las TPF con cuatro variables socioeconómicas básicas representativas del nivel de desarrollo de una región: la pobreza, la desigualdad, la marginación y el desarrollo humano, así como con algunos indicadores específicos de género. En la gráfica 7 podemos observar la relación inversa entre TPF y los niveles de pobreza a escala municipal. Para medir la pobreza utilizamos los cálculos del indicador $\mathrm{FGT}_{2}$ para los municipios de Chihuahua elabo- 
Gráfica 6.

Relación entre estructura económica y tasas de participación femenina.

Chihuahua, 1990-2000

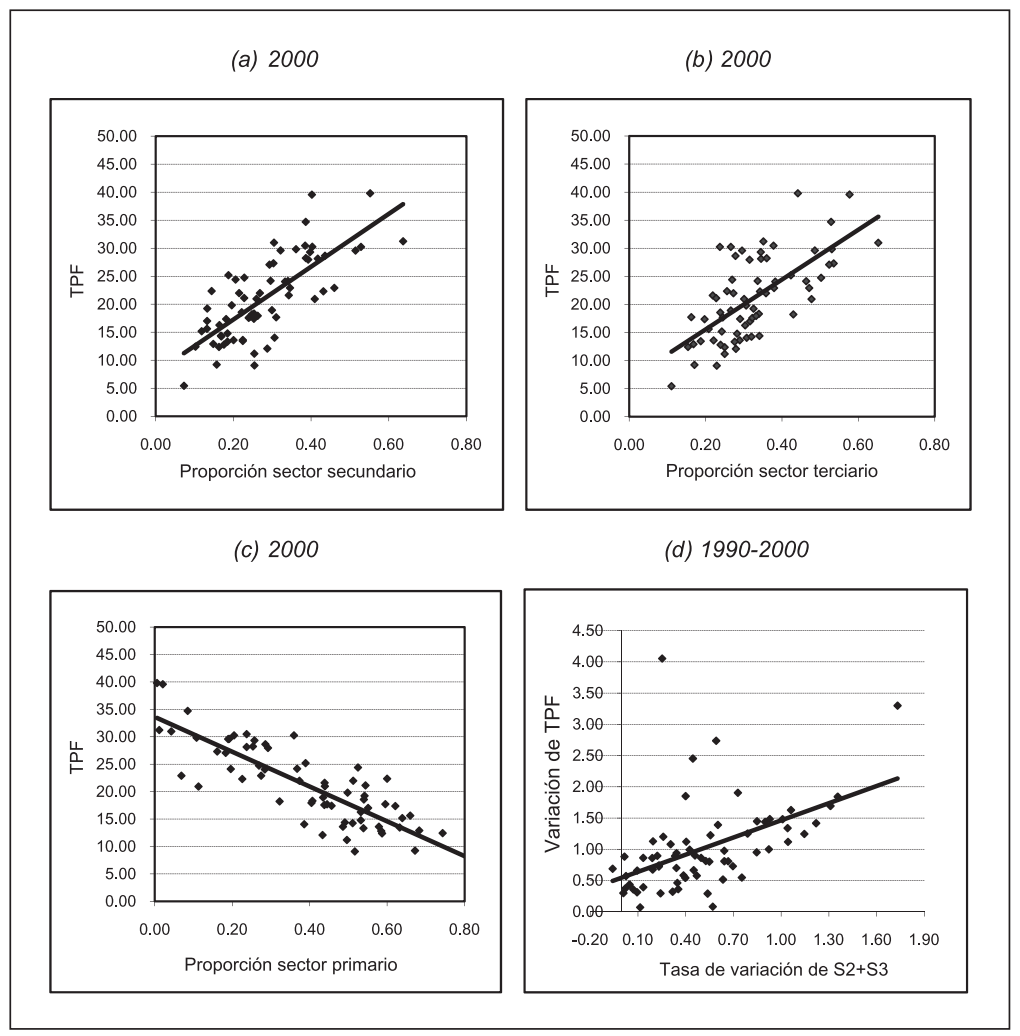

Fuente: Elaboración propia con base en INEGI $(1990,2000)$.

rados en otros estudios sobre la pobreza de ingreso en la entidad (Gutiérrez, 2006a)..$^{13}$ La relación TPF y pobreza se confirma tanto en 1990 como en 2000, por lo que es posible afirmar que mayores tasas de participación femenina están asociadas a una menor pobreza de la

${ }^{13} \mathrm{El}$ indicador $\mathrm{FGT}_{2}$ (Foster, Green y Thorbecke) se entiende como una medida de la severidad de la pobreza e incluye la intensidad y la brecha de la pobreza. 


\section{Gráfica 7.}

Pobreza de ingreso y tasas de participación femenina por municipio

Chihuahua, 1990-2000

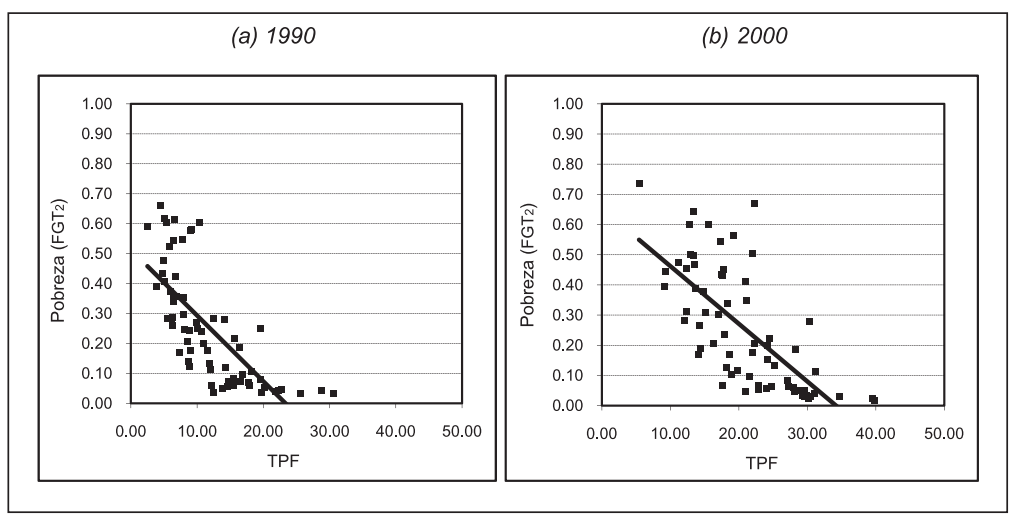

Fuente: Elaboración propia con base en Gutiérrez (2006a) e INEGI (1990, 2000).

población. Asimismo, en la gráfica 8 se representa la relación entre desigualdad de ingreso -calculada mediante el índice de Gini [IGI]- y la participación femenina en los mercados de trabajo. ${ }^{14}$

La relación es también inversa y muestra un vínculo relevante: según los resultados de este ejercicio, una mayor incorporación de la población femenina al mercado de trabajo ayuda a aminorar los problemas de desigualdad de ingreso en la región.

En cuanto al grado de marginación, éste también disminuye cuando hay tasas de participación femenina más elevadas. Si utilizamos el índice de marginación [IMA] que el Conapo construyó para el año 2000, obtenemos la gráfica 9a que arroja una relación inversa entre TPF y grado de marginación. En lo general, el nivel de desarrollo -considerando también las dimensiones de educación y salud- se encuentra asociado a una incorporación más intensa de la mujer al mercado de

${ }^{14}$ En los casos de la asociación entre los indicadores socioeconómicos y la TPF, ubicamos a esta última como variable explicativa conforme al marco de relaciones causales expuesto en el apartado siguiente. 
Gráfica 8.

Desigualdad de ingreso y tasas de participación femenina por municipio.

Chihuahua, 1990-2000

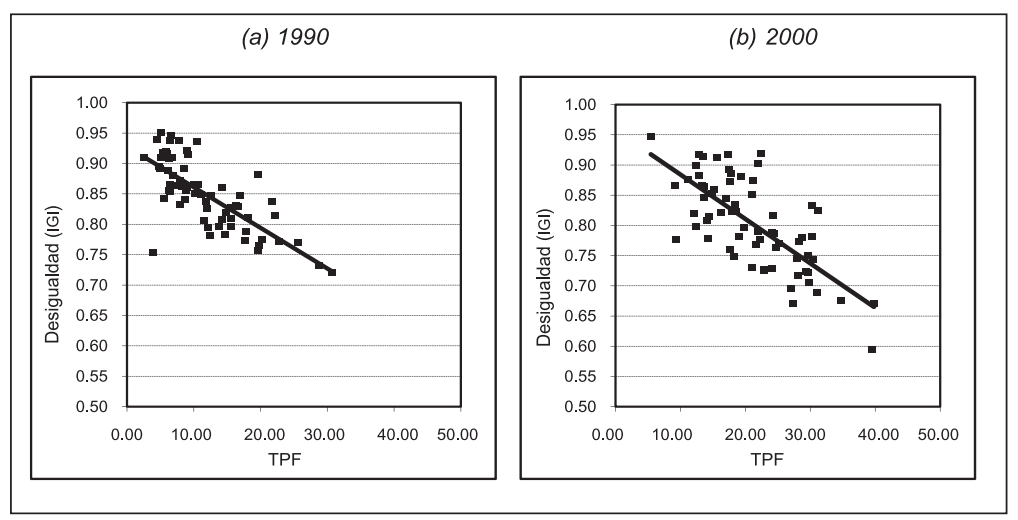

Fuente: Elaboración propia con base en Gutiérrez (2007) e INEGI (1990, 2000).

Gráfica 9.

Marginación, desarrollo humano y tasas de participación femenina por municipio.

Chihuahua, 2000

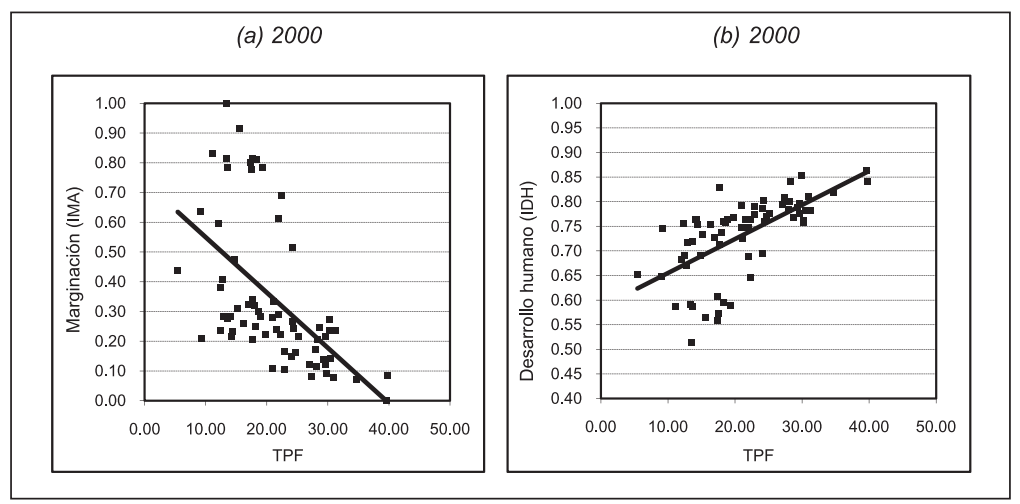

Fuente: Elaboración propia con base en Conapo (2001a, 2001b) e INEGI (2000). 
Mapa 1.

Tasas de participación femenina y nivel socioeconómico por municipio.

Chihuahua, 2000

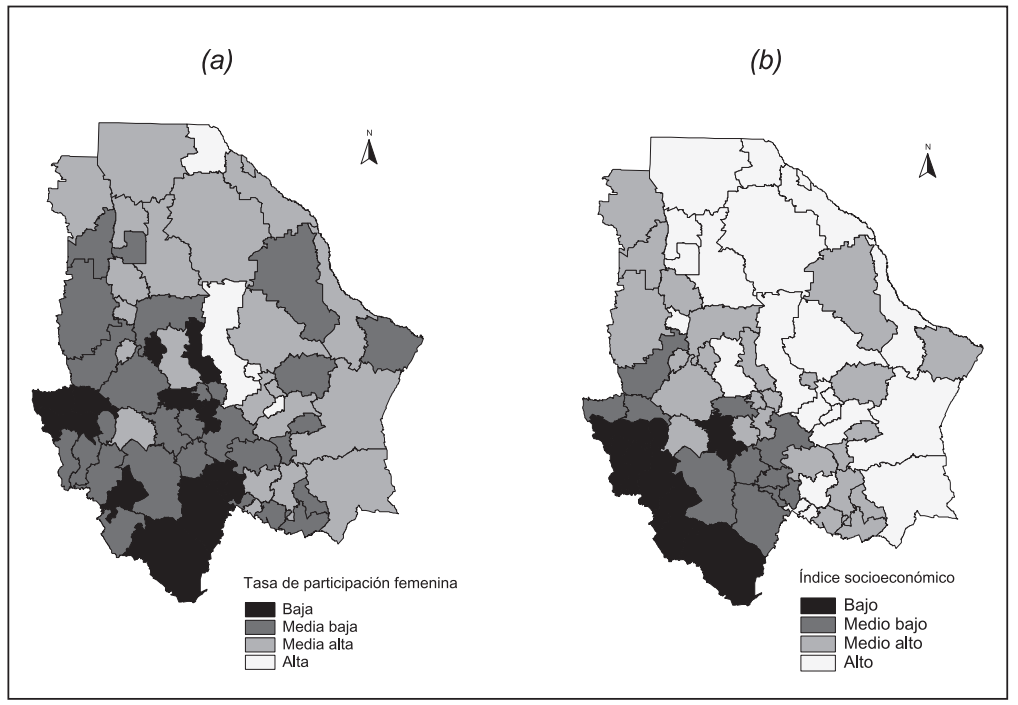

Fuente: Elaboración propia con base en CONAPO (2001a, 2001b) e INEGI (1990, 2000).

trabajo. La gráfica $9 \mathrm{~b}$ constata esta relación. En ella se utiliza el índice de desarrollo humano [IDH] y se aprecia una relación directa entre TPF e IDH. Para ofrecer una perspectiva territorial de algunos vínculos examinados, presentamos el mapa 1 en el que se observa la asociación entre las variables de corte socioeconómico (con base en un indicador compuesto, el índice socioeconómico -ISO-, que agrupa los indicadores de pobreza, desigualdad, marginación y desarrollo humano) y las tasas de participación femenina. ${ }^{15}$ Conforme al mapa 1, las regiones sur y suroeste del estado muestran las menores TPF y un nivel de desarro1 lo más bajo.

${ }^{15}$ El índice socioeconómico que utilizamos se obtiene mediante la ecuación:

$$
\mathrm{ISO}=\left[\mathrm{FGT}_{2}+\mathrm{IGI}+\mathrm{IMA}+(1-\mathrm{IDH}] / 4\right.
$$


Gráfica 10.

Indicadores de género y tasas de participación femenina por municipio.

Chihuahua, 2000

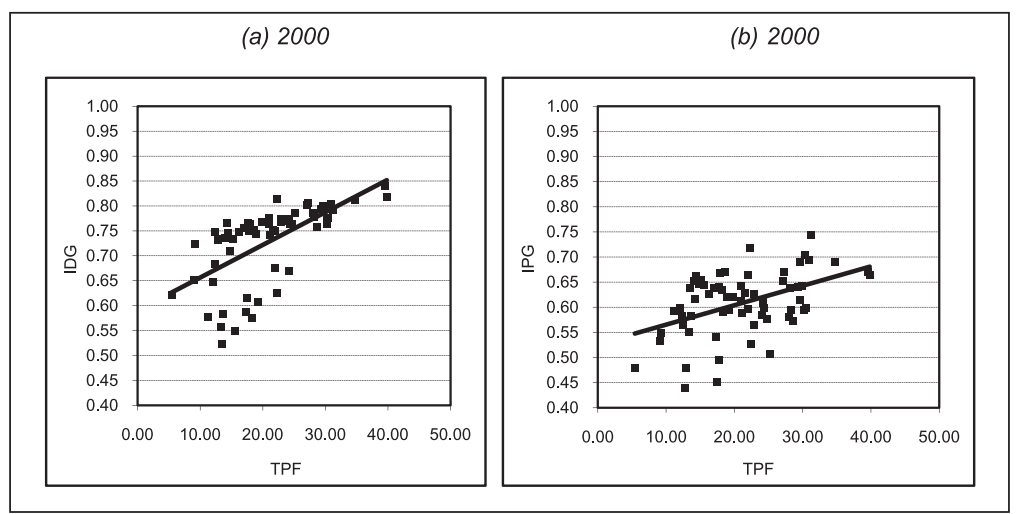

Fuente: Elaboración propia con base en PNUD (2006) e INEGI (2000).

Nota: Para los municipios de Dr. Belisario Domínguez y Urique no hay información disponible.

Si examinamos el comportamiento que tienen otros indicadores del desarrollo que de manera explícita hacen referencia a las desigualdades de género y los comparamos con las TPF, también podemos encontrar asociaciones importantes. Es el caso del índice de desarrollo relativo al género [IDG] y el índice de potenciación de género [IPG]. El primero hace referencia a la desigualdad de género en las dimensiones de educación, salud e ingreso (PNUD, 2006). ${ }^{16}$ En la medida que se presentan mayores diferencias entre hombres y mujeres en estos tres rubros, el IDG tiende a cero. En el caso de Chihuahua, una mayor equidad entre hombres y mujeres se encuentra asociada directamente a las tasas de participación femenina (gráfica 10a).

16 Una crítica al IDG puede leerse en Dijkstra y Hanmer (2000), quienes proponen un nuevo indicador: el índice del estatus relativo de la mujer (RSW, por sus siglas en inglés). 
El IPG, por su parte, expresa la desigualdad de género en los ámbitos de la participación y el poder de decisión política y económica, así como en el nivel de ingreso. En el mismo sentido que el IDG, a medida que el índice de potenciación de género tiende a cero se representa una mayor desigualdad. Como se revela en la gráfica 10b, un aumento en las TPF en los municipios de Chihuahua conduce a un incremento del IPG, por lo que la relación es directa, pese a que se percibe una mayor dispersión de los datos. No obstante, podemos concluir que las participación de la mujer en la economía se asocia no únicamente a variables estrictamente económicas, sino a otras de carácter político también relacionadas con el desarrollo.

\section{Una interpretación sobre las relaciones de causalidad entre la feminización y el desarrollo regional}

En principio, como sugerimos al inicio de este ensayo, entendemos que hay una relación causal "interactiva" entre la participación de la mujer en el mercado laboral y el desarrollo. En el caso del estado fronterizo de Chihuahua se ha hecho patente este vínculo, expresado en la estrecha relación entre la TPF y la estructura económica, el nivel de ingreso, la pobreza, la desigualdad, la marginación y el desarrollo humano. En este sentido, podemos plantear un marco explicativo del desarrollo regional en el que la incorporación de la mujer al ámbito productivo desempeña un papel relevante.

Como se propone en el diagrama 2, el cambio en la estructura productiva es un punto de partida práctico para entender las variaciones en las TPF y sus consecuencias. En tanto una región abandona las actividades agropecuarias atrasadas y menos rentables para dar paso a procesos de industrialización y terciarización más avanzados, se constata una mayor participación de la mujer en el mercado de trabajo. Aun cuando en estudios de otras regiones se ha centrado la atención en la expansión del sector terciario para explicar los incrementos en las TPF, en el caso de Chihuahua es necesario examinar el crecimiento de los sectores secundario y terciario como conjunto. De hecho, el papel preponderante que tiene la industria maquiladora en el estado -en particular en los municipios más grandes de la entidad- con- 


\section{Diagrama 2.}

Relaciones de causalidad entre el desarrollo regional y la participación de la mujer en el mercado de trabajo

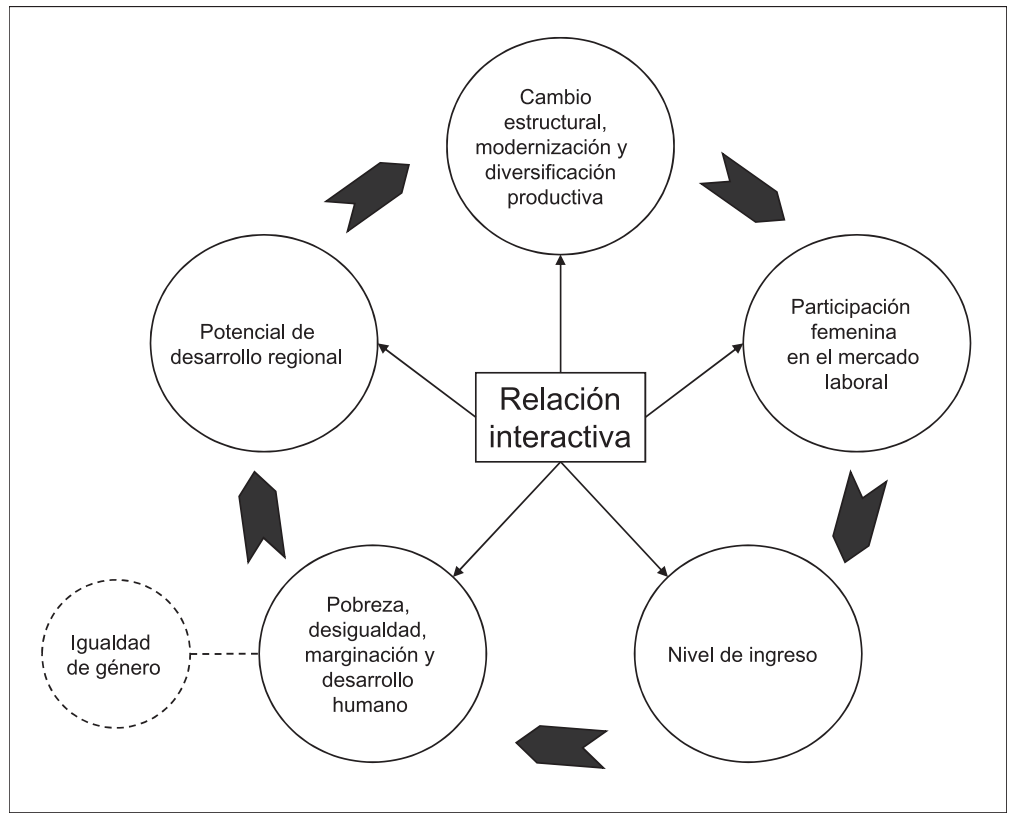

Elaboración propia.

lleva la inclusión de la actividad secundaria como factor explicativo de los cambios en la participación femenina dentro de la economía regional. ${ }^{17}$

Por otra parte, es lógico que cuando un importante segmento de la población entra por primera vez al mercado laboral y se emplea -en este caso las mujeres-, el nivel de ingreso per cápita de los municipios también se incrementa, lo que favorece el potencial de mercado y el bienestar de la población en general. En Chihuahua la relación entre las TPF y el nivel de ingreso ha sido positiva, conforme se vio en los apartados anteriores.

${ }^{17}$ Momsen (2006) explica que la economía internacional, mediante la articulación de empresas manufactureras transnacionales, ha influido en la participación femenina mediante la creación de nuevos mercados laborales para la mujer. 
Asimismo, en el caso del estado de Chihuahua se observó que a partir de que las TPF y el nivel de ingreso se incrementan, los indicadores de pobreza y desigualdad tienden a mostrar una tendencia a disminuir, en tanto que el índice de desarrollo humano aumenta. La asociación entre TPF y el desarrollo es más clara cuando se le relaciona con los indicadores de género que miden el grado de desigualdad en el nivel de desarrollo de hombres y mujeres. Como se vio anteriormente, los municipios con mayores TPF resultan en un IDG y un IPG superiores, y por lo tanto con una menor desigualdad de género, lo que implica un nivel de desarrollo más elevado para las mujeres.

Por consiguiente, la mejoría en el nivel de ingreso y en los indicadores socioeconómicos de pobreza, desigualdad y marginación propician cambios positivos en la capacidad de las regiones para responder a las transformaciones del entorno y adaptar su estructura productiva. Un trabajo ya mencionado como el de Gutiérrez (2007) ha demostrado que la reducción de la pobreza y la desigualdad conllevan un aumento del potencial regional de desarrollo [PRD]. ${ }^{18}$ Desde esta perspectiva se puede afirmar, de acuerdo a la experiencia específica de Chihuahua, que una participación más amplia de la mujer en el ámbito productivo genera un mayor potencial de desarrollo para las regiones.

Para constatar estadísticamente las correlaciones descritas anteriormente -expuestas en el diagrama 2-, se hizo un ejercicio de regresión en el que se examinó, a escala municipal, la relación entre la estructura económica [EST] y las TPF, por una parte; las TPF y el nivel de ingreso [IPC], por otra; el IPC y los indicadores socioeconómicos de pobreza, desigualdad, marginación y desarrollo humano; y, finalmente, las TPF con el IDG y el potencial regional de desarrollo [PRD]. Los resultados del ejercicio se exponen en el cuadro 1. Cabe decir que en cada caso se confirmaron los vínculos que de manera reiterada se han mencionado a lo largo del trabajo. ${ }^{19}$ De acuerdo con los resultados de este cuadro,

\footnotetext{
${ }^{18}$ Según el trabajo de Gutiérrez, la reducción en los niveles de pobreza, marginación y desigualdad reduce los factores limitantes del desarrollo de las regiones y permite a éstas aprovechar en forma más óptima y eficiente los recursos que potencian el desarrollo.

${ }^{19}$ El objetivo del este ejercicio es validar los vínculos entre las TPF y el desarrollo de una región. No obstante, se parte del supuesto de una relación "interactiva" entre las variables.
} 
Cuadro 1. Relaciones entre la participación femenina en el mercado laboral y el desarrollo regional.

Mediciones estadísticas

\begin{tabular}{ccccccc}
\hline $\begin{array}{c}\text { Variable } \\
\text { explicada }\end{array}$ & C & EST & TPF & IPC & $\mathrm{R}^{2}$ & $\mathrm{~N}$ \\
\hline \multirow{2}{*}{ TPF } & 1.855013 & 31.48788 & & & 0.72 & 64 \\
& $(1.579464)$ & $(2.391258)$ & & & D.W. 1.95 & \\
IPC & -0.100171 & & 0.017894 & & 0.60 & 64 \\
& $(0.041214)$ & & $(0.001881)$ & & D.W. 1.98 & \\
ISO & 0.594577 & & & -0.631435 & 0.58 & 64 \\
& $(0.023064)$ & & & $(0.067693)$ & D.W. 1.94 & \\
IDG & 0.587644 & & 0.006657 & & 0.40 & 62 \\
& $(0.023650)$ & & $(0.001052)$ & & D.W. 1.95 & \\
PRD & -0.110943 & & 0.020491 & & 0.67 & 67 \\
& $(0.039962)$ & & $(0.001816)$ & & D.W. 1.99 & \\
& & & & & & \\
\hline
\end{tabular}

* $\quad$ Entre paréntesis el error estándar.

** $\quad$ La $R^{2}$ y la D.W. son cifras redondeadas.

Nota: En los tres primeros casos no se consideraron los municipios de Galeana, Gómez Farías y Matachí. En el cuarto, además de los tres municipios anteriores, no se incluyeron Dr. Belisario Domínguez y Urique.

Fuente: Elaboración propia con base en Conapo (2001a, 2001b), INEGI (1990, 2000), PNUD (2006) y Gutiérrez (2006a).

se puede apreciar que el nivel explicativo entre tasas de participación femenina y el ingreso per cápita, los rasgos socioeconómicos y el potencial regional de desarrollo (se alcanza una $\mathrm{R}^{2}$ de $0.72,0.58$ y 0.67 , respectivamente), es significativo para plantear una asociación causaefecto entre incorporación de la mujer al mercado de trabajo y nivel de desarrollo.

Por último, debe anotarse que las relaciones entre las TPF y el desarrollo, conforme se esquematizan en el diagrama 2 y con base en los resultados del cuadro 1, sólo intentan mostrar el papel significativo que tiene la mujer como agente del desarrollo al incorporarse al mercado de trabajo. Obviamente, los factores causales del crecimiento y el desarrollo regional conforman un abanico mucho más amplio y complejo (Gutiérrez, 2006b). No obstante, es el grado de asociación entre 
las TPF y las variables del desarrollo lo que nos interesa resaltar en este ensayo. ${ }^{20}$

\section{Conclusiones}

A lo largo de este ensayo se expusieron una serie de vínculos entre el proceso de desarrollo regional y la incorporación de la mujer a la actividad productiva utilizando el caso de los municipios del estado de Chihuahua. En suma, las diferentes variables representativas del desarrollo se mostraron sensibles a los cambios en las tasas de participación femenina. Como resultado general de las mediciones, la relación entre los indicadores de la estructura productiva, el ingreso y el nivel socioeconómico respecto a las TPF resultó positiva; más aún, en el contexto de la discusión sobre el comportamiento de las tasas de participación femenina según el grado de desarrollo, los municipios de Chihuahua no confirmaron la hipótesis de la curva $U$ planteada en el primer apartado. Si se considera al conjunto de relaciones entre variables socioeconómicas y participación femenina, el comportamiento de las TPF se mostró prácticamente lineal (ver, por ejemplo, la gráfica 4).

Por otra parte, el examen de la asociación entre TPF y desarrollo en Chihuahua nos permitió construir un marco analítico que supone una serie de relaciones causales "interactivas" en el que la entrada de la mujer al mercado laboral desempeña un papel significativo. Al respecto se hizo la aclaración de que el mismo desarrollo de las regiones es un proceso más complejo en el que intervienen un sinnúmero de variables. No obstante, en este trabajo, considerando los resultados obtenidos, se resaltó el papel de la participación femenina en el desarrollo -incremento del ingreso y reducción de la pobreza y la desigualdad- y su importante aportación a las economías regionales.

En este sentido, es pertinente agregar que desde la perspectiva de las políticas públicas se debe impulsar la participación de la mujer en

${ }^{20}$ Cabe anotar, como ya se ha observado a lo largo del trabajo, que se trata de un ejercicio de corte transversal, por lo que las relaciones de causalidad que se plantean se hacen bajo las restricciones propias de un análisis empírico de esta naturaleza. 
la economía y propiciar cambios sociales y materiales que favorezcan su incorporación al ámbito productivo y eliminen los obstáculos que no permiten aprovechar de manera óptima su capacidad potencial. ${ }^{21}$ Incluso, desde la perspectiva de género, también es necesario tener en cuenta que la entrada de la mujer al mercado laboral le ha propiciado situaciones negativas de desigualdad, inseguridad y precariedad dentro de los centros de trabajo, por lo que es preciso que estos problemas se incluyan en la agenda de obstáculos a superar por las políticas de desarrollo. ${ }^{22}$ Bajo este entendido, las políticas de género dirigidas a la formación, igualdad y seguridad deben considerarse dentro de las estrategias de desarrollo e incorporarse, como dice Astelarra (2005), a la maquinaria institucional, en este caso de las regiones.

Albert Hirschman (1961) escribió que "el desarrollo no depende tanto de saber encontrar las combinaciones óptimas de recursos y factores de producción dados como de conseguir, para propósitos de desarrollo, aquellos recursos y capacidades que se encuentren ocultos, diseminados o mal utilizados". Bajo esta premisa es evidente que la mujer es un recurso humano fundamental y un agente esencial del desarrollo que poco a poco ha develado su amplia capacidad productiva y su importante aportación a la economía de las regiones. En este sentido, en la medida en que se formulen y apliquen políticas que fomenten la formación de la mujer y se genere un ambiente propicio para el desarrollo de sus capacidades, necesariamente se estará contribuyendo a mejorar el nivel de vida de la población en general, lo que a fin de cuentas es el objetivo de toda política de desarrollo.

${ }^{21}$ Ruth Pearson escribe que "las mujeres participan con su tiempo, energía, trabajo y habilidades en todos los aspectos del proceso de desarrollo; es la desigualdad de las relaciones de género y la persistente subordinación de las mujeres lo que determina que su aportación no tenga la debida contrapartida, en forma de reconocimiento y remuneración, en el aspecto social, político y económico" (Pearson, 2003:389).

22 Autoras como Fall (2003) exponen el problema de la feminización de la pobre$\mathrm{za}$, el cual se ha agudizado en países menos desarrollados. 


\section{Bibliografía}

Abramo, Laís y María Elena Valenzuela (2005), “Women’s labour force participation rates in Latin America", International Labour Review, 144 (4): 369-399.

Astelarra, Judith (2005), Veinte años de políticas de igualdad, España, Cátedra, Colección Feminismos, Universitat de Valencia, Instituto de la Mujer.

Barker, Drucilla (2005), “Beyond women and economics: rereading women's work", Journal of Women in Culture and Society, 30 (4): 2189-2209.

Benería, Lourdes (1981), "Conceptualizing the labor force: the underestimation of women's economic activities", Journal of Development Studies, 17 (3): 10-27.

(2003a), “La mujer y el género en la economía. Un panorama general”, en Paloma de Villota (ed.), Economía y género, España: Icaria, Economía. (2003b), Género, desarrollo y globalización, España, Hacer Editorial.

Bose, Christine E. (1987), “Devaluing women's work”, en Christine Bose et al., Hidden aspects of women's, Estados Unidos, Praeger.

Boserup, Esther (1970), Women's role in economic development, Estados Unidos, St. Martin's Press, 283 p.

Consejo Nacional de Población (2001a), Índices de desarrollo humano, 2000, México, CONAPO. (2001b), Índices de marginación, 2000, México, CONAPO.

Dijkstra, A. y Lucía Hanmer (2000), "Measuring socio-economic gender inequality: toward an alternative to the UNDP gender related development index", Feminist Economics, 6 (2): 41-75.

Dureya, Suzanne, Alejandra Cox, et al. (2001), Women in latinamerican labor market: the remarkable 1990's, ponencia presentada en el Primer Seminario Técnico de Consulta Regional sobre Temas Laborales, Panamá.

Fall, Yassine (2003), “Género y pobreza”, en Paloma de Villota (ed.), Economía y Género, pp. 111-128, España, Icaria.

Gutiérrez C., Luis E. (2006a), “Pobreza de ingreso en Chihuahua. Un análisis territorial para el período 1990-2000”, en Víctor Orozco 
(coord.), Chihuahua Hoy, tomo IV, pp. 129-158, México, Instituto Chihuahuense de la Cultura, Universidad Autónoma de Ciudad Juárez.

(2006b), “Teorías del crecimiento regional y el desarrollo divergente", Nóesis, 15 (30): 185-227.

(2007), "Potencial de desarrollo y gestión de la política regional: el caso de Chihuahua", Frontera Norte, 19 (38): 7-35, México.

Hirschman, Albert O. (1961), La estrategia del desarrollo económico, México, Fondo de Cultura Económica.

Instituto Nacional de Estadística Geografía e Informática (1990), XI Censo Nacional de Población y Vivienda, México, INEGI. (2000), XII Censo Nacional de Población y Vivienda, México, INEGI. Jacobsen, Joyce (1998), The economics of gender, Estados Unidos, Blackwell.

Kuznets, Simon (1970), Crecimiento económico y estructura económica, España, Gustavo Gili, Colección de Ciencia Económica.

Loutfi, Martha F. (2001), Women, gender and work: what is equality and how do we get there?, Suiza, Organización Internacional del Trabajo.

Mammen, Kristin y Christina Paxson (2000), “Women's work and economic development", Journal of Economic Perspectives, 14 (4): 141-164.

Martínez Quintana, Violante (2001), Estudios y políticas sociales para las mujeres, España, Universidad Nacional de Educación a Distancia, Programa de Enseñanza Abierta, Colección Educación Permanente.

Maxwell, Nan (1990), "Changing labor force participation: influences on income inequality and distribution", Social Forces, 68 (4): 12511266.

Michael, Robert (1985), "Consequences of the rise in female labor force participation rates: questions and probes", Journal of Labor Economics, 3 (1) S117-S146.

Momsen, Janet (2006), Gender and development, Estados Unidos, Routledge.

Pampel, Fred y Kazuko Tanaka (1986), “Economic development and female labor force participation: a reconsideration", Social Forces, 64 (3): 599-619. 
Pearson, Ruth (2003), "El género cuenta en el desarrollo", en Cristina Carrasco (coord.), Mujeres y economía. Nuevas perspectivas para nuevos y viejos problemas, España, Icaria.

Pickford S., Fredricka (1976), "The role of women in the development process: market integration or family disintegration", Journal of International Affairs, 30 (2): 173-181.

PNUD (2006), Indicadores de desarrollo humano y género en México, México, Programa de las Naciones Unidas para el Desarrollo.

Rau, William y Robert Wazienski (1999), “Industrialization, female labor force participation, and the modern division of labor by sex", Industrial Relations, 38 (4): 504-521.

Ray, Debraj (1998), Economía del desarrollo, España, Antoni Bosch.

Schultz, Paul (1990), "Women's changing participation in the labor force: a world perspective", Economic Development and Cultural Change, 38 (3): 457-488.

Seguino, Stephanie y Caren Grown (2006), Feminist-Kaleckian macroeconomic policy for developing countries, Cuaderno de trabajo, 446, Estados Unidos, The Levy Economics Institute of Bard College. Shorter, Edward (1973), "Female emancipation, birth control, and fertility in european history", American Historical Review, 78: 605640.

Smith, James y Michael Ward (1989), "Women in the labor market and in the family", Journal of Economic Perspectives, 3 (1): 9-23.

Vara Miranda, María Jesús (2006), “Empleo femenino en las cadenas de producción global”, en María J. Vara (coord.), Estudios sobre Género y Economía, España, Akal, Economía Actual.

Recibido en octubre de 2007

Segunda versión recibida en mayo de 2008

Aprobado en agosto de 2008 


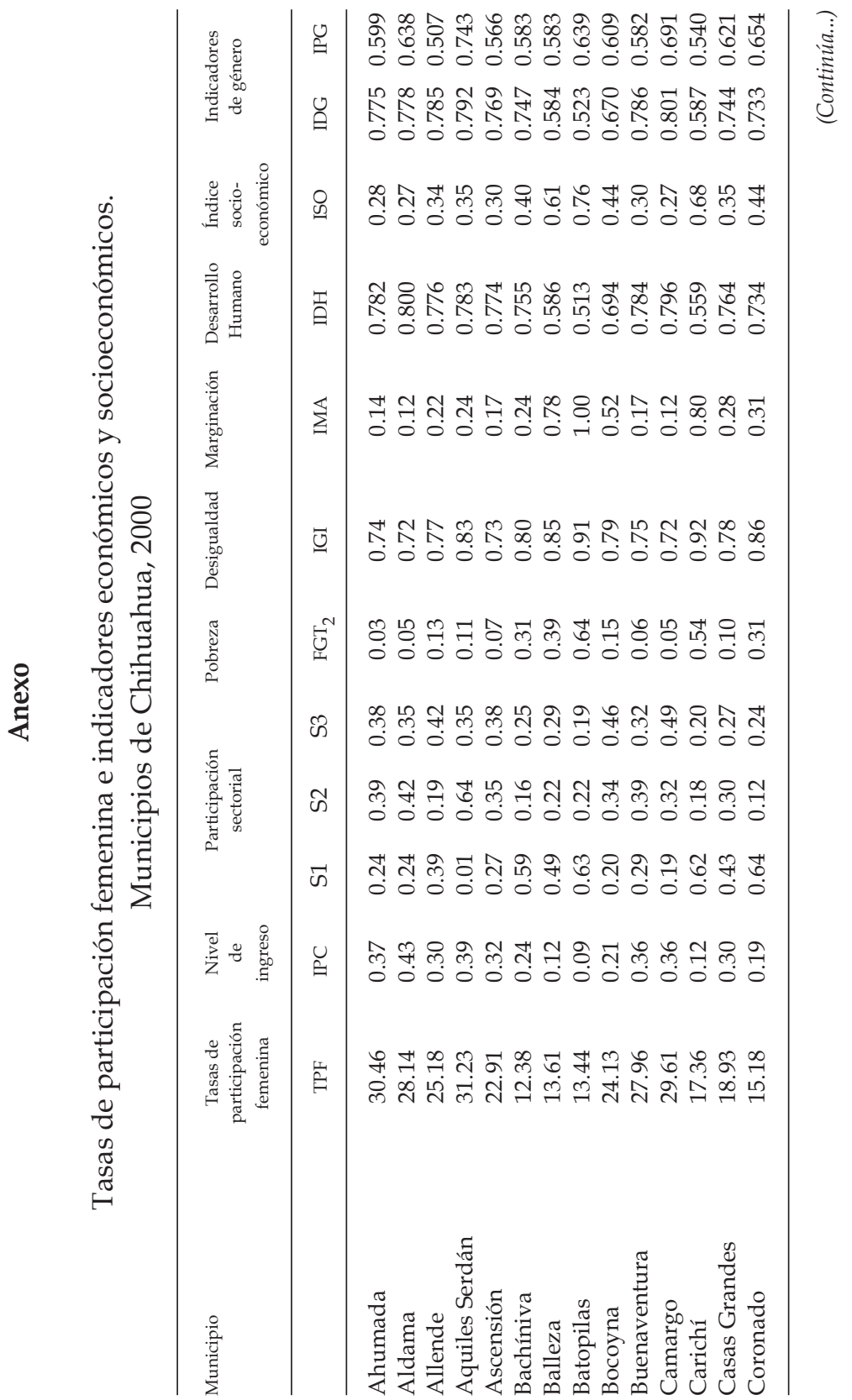




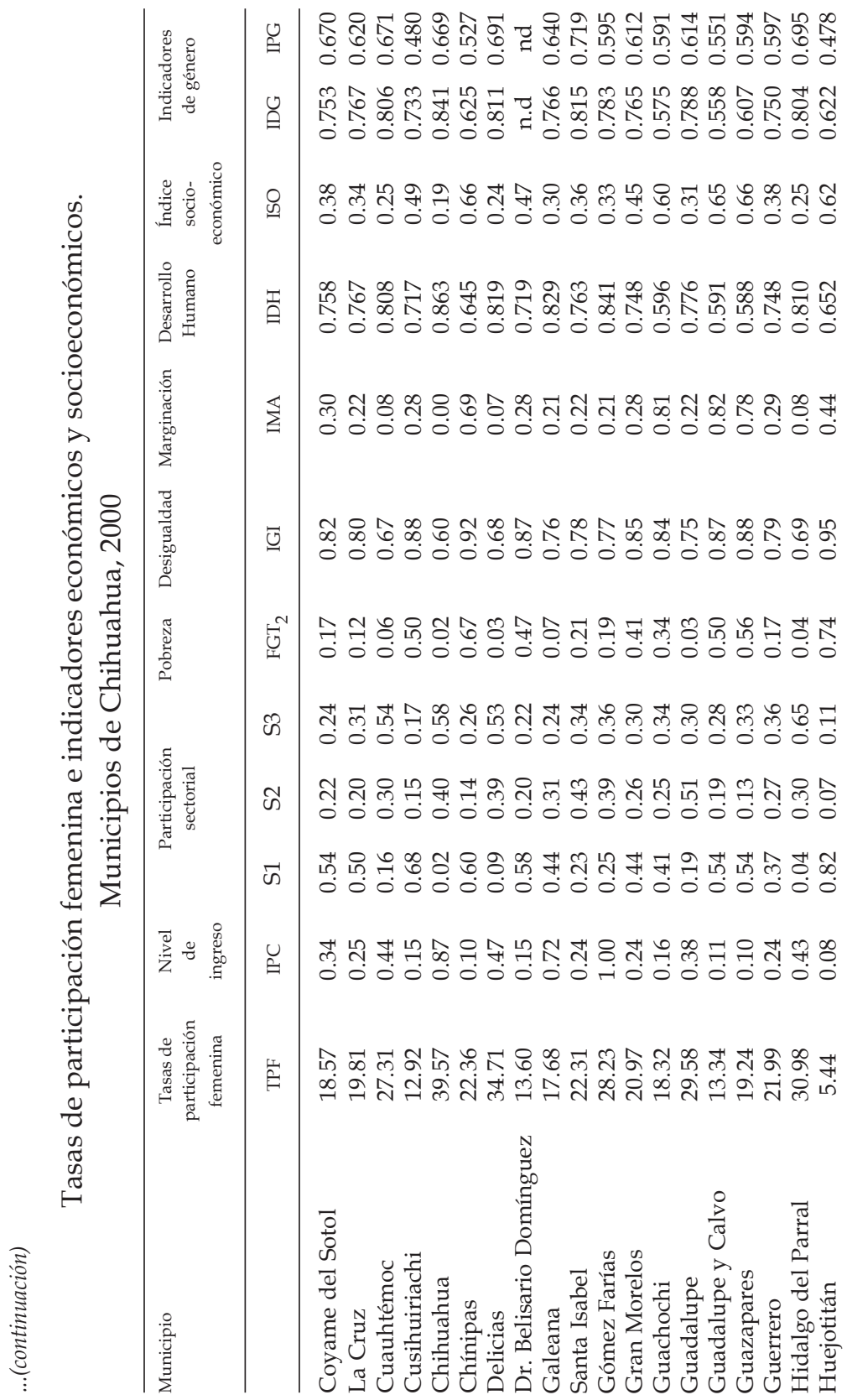




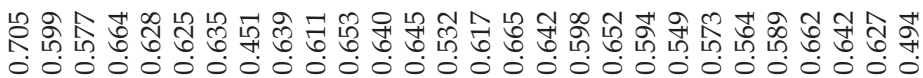

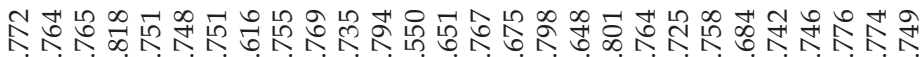

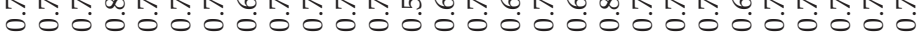

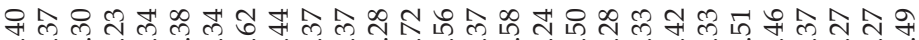
000000000000000000000000

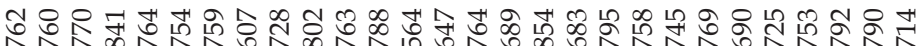

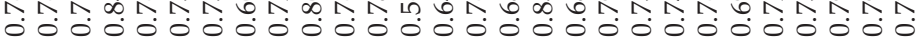

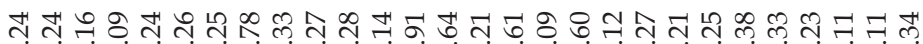
0000000000000000000000000000

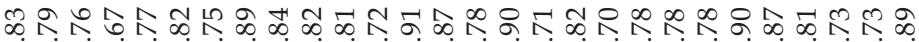

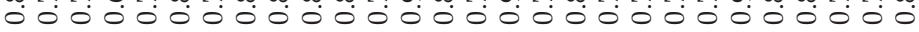

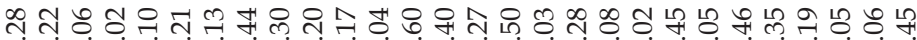

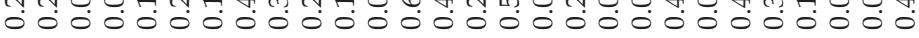

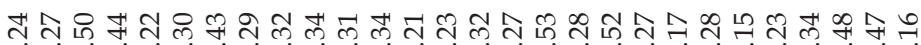
00000000000000000000000000000

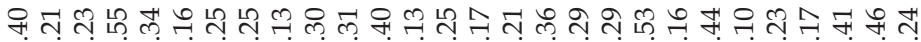

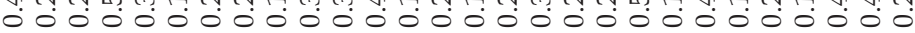

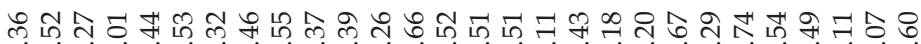
0000000000000000000000000

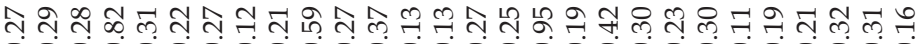
00000000000000000000000000

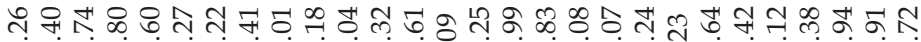

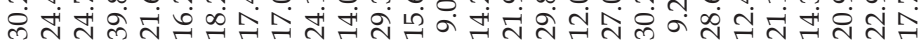

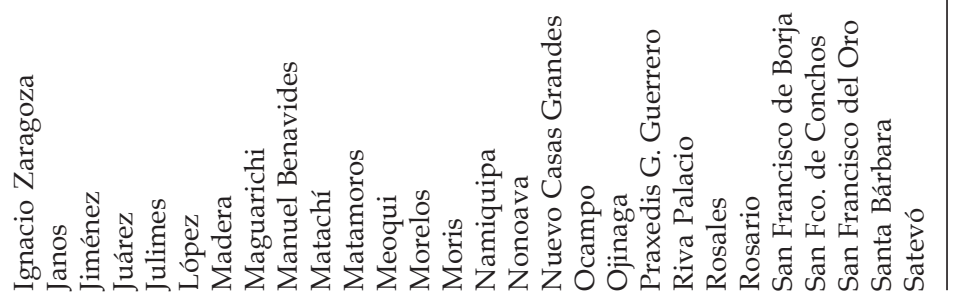




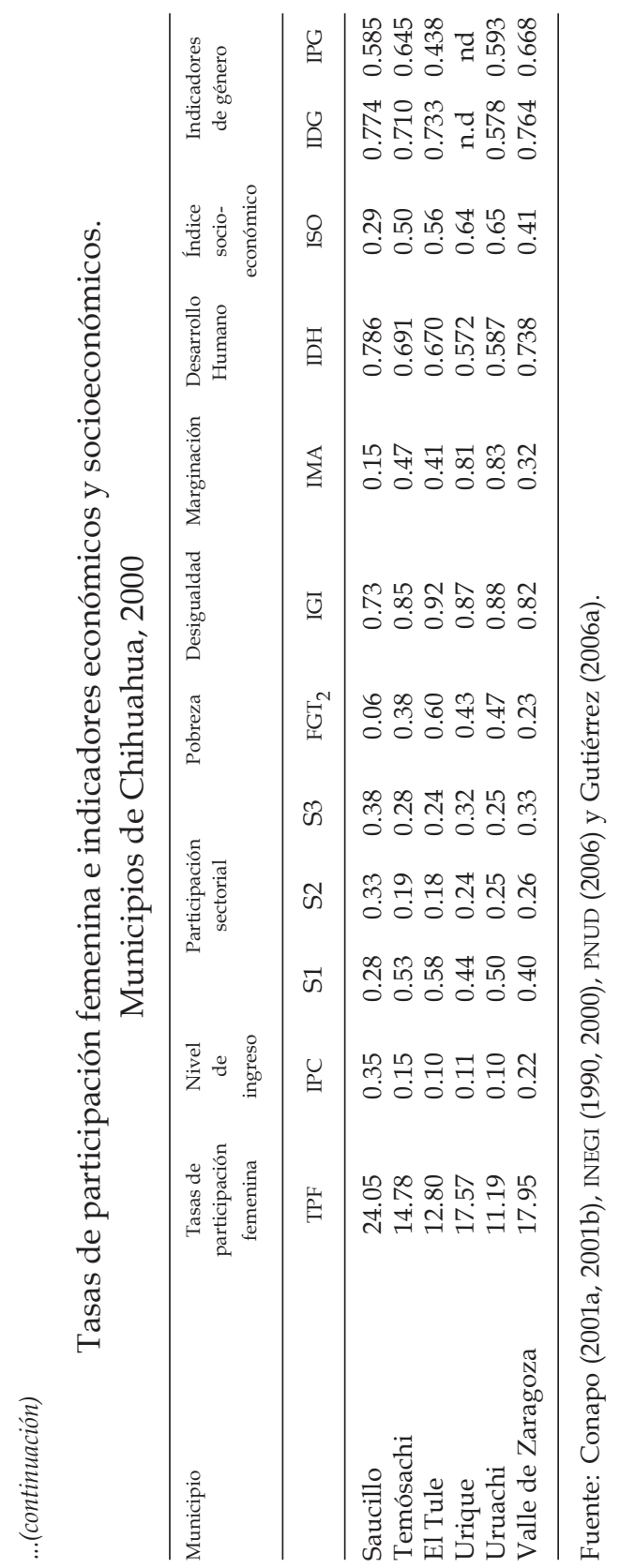

